\title{
ANÁLISE DOS ÓBITOS EVITÁVEIS DE MENORES DE CINCO ANOS NO MUNICÍPIO DE MARINGÁ-PR ${ }^{a}$
}

\author{
Analysis of preventable deaths in children under five years in Maringá-PR \\ Análisis de los óbitos evitables en menores de cinco años en Maringá-PR
}

Denise Albieri Jodas ${ }^{1}$

Maria José Scochi

Jessica Batistela Vicente ${ }^{3}$

Andréa Guimarães Colucci ${ }^{4}$

\section{RESUMO}

0 objetivo deste estudo foi analisar os óbitos de menores de cinco anos por doenças evitáveis ocorridos em 2008 no município de Maringá, PR. Estudo quantitativo, descritivo, com 41 óbitos. Como instrumento norteador de coleta de dados, utilizou-se 0 formulário de investigação do Comitê de Prevenção do Óbito Infantil e Fetal. Dos 41 casos encontrados, 65,9\% representaram óbitos neonatais, $24,4 \%$ pós-neonatais e $9,7 \%$, de criança com mais de um ano; destes, $90,2 \%$ óbitos foram considerados evitáveis, sendo $64,9 \%$ neonatais, $27,0 \%$ pós-neonatais e $8,1 \%$ na idade acima de um ano. Quanto às medidas de evitabilidade, percebeu-se que as atividades de prevenção foram as mais encontradas entre as possibilidades de redução do óbito, com 59,5\%, seguidas de ações de diagnóstico e tratamento precisos, com 35,1\%. Conclui-se que a maioria dos óbitos evitáveis tem relação direta com a assistência à gestante no pré-natal e no parto, demonstrando a necessidade de instituir uma prática efetiva e de qualidade.

Palavras-chave: Atenção primária à saúde. Avaliação em saúde. Vigilância de evento sentinela.

\begin{abstract}
Analyze the deaths in children under five years old, from preventable diseases have occurred in 2008 in Maringá, PR. Quantitative and descriptive study, with 41 deaths. As a guiding instrument for data collection, we used the form of research of the Committee on the Prevention of Infant and Fetal Death. From the 41 cases found, neonatal deaths accounted for $65.9 \%$, $24.4 \%$ post-neonatal deaths and $9.7 \%$ above a year, $90.2 \%$ of these deaths were considered preventable, being $64.9 \%$ neonatal, $27.0 \%$ post-neonatal and $8.1 \%$ newborn after one year old. For measures of preventability, it was realized that prevention activities were found more often among the possibilities for reducing death, with $59.5 \%$, followed by actions of accurate diagnosis and treatment with $35.1 \%$. It was concluded that the majority of preventable deaths has direct relationship with the care of pregnant women in prenatal and childbirth, demonstrating the need to establish an effective practice and quality.
\end{abstract}

Keywords: Primary health care. Health evaluation. Sentinel Surveillance.

\section{Resumen}

Analizar las muertes de niños menores de cinco años por enfermedades evitables ocurridas en 2008 en Maringá, PR. Investigación cuantitativa, descriptiva, con 41 óbitos. Como instrumento de orientación para la recolección de datos, se utilizó el formulario de investigación de la Comisión de la Prevención de la Mortalidad Infantil y Fetal. De los 41 casos encontrados, un $65,9 \%$ representaron óbitos neonatales, $24,4 \%$ post-neonatales y $9,7 \%$ por encima de un año; de estos $90,2 \%$ de las muertes fueron consideradas evitables, siendo un $64,9 \%$ neonatales, $27,0 \%$ post-neonatales y $8,1 \%$ en la edad por encima de un año. Cuanto a las medidas de prevención, se observó que las actividades de prevención se han encontrado con mayor frecuencia entre las posibilidades de reducción de la muerte, dejando a un $59,5 \%$, seguido por las acciones de diagnóstico y tratamiento, con un $35,1 \%$. Conclusión: la mayoría de las muertes evitables tiene relación directa con el cuidado de las mujeres embarazadas en control prenatal y el momento del parto, lo que demuestra la necesidad de establecer una práctica efectiva y de calidad.

Palabras clave: Atención Primaria de Salud. Evaluación en Salud. Vigilancia de Guardia

\footnotetext{
${ }^{1}$ Mestre em Enfermagem pela Universidade Estadual de Maringá-UEM. Docente do curso Técnico em Enfermagem do Instituto Federal do Paraná, campus Londrina. Londrina - PR. Brasil. E-mail: denisealbieri@yahoo.com.br; ${ }^{2}$ Doutora em Saúde Publica. Orientadora. Docente da Universidade Estadual de Maringá - UEM. Maringá - PR. Brasil. E-mail: mjscochi@uem.br; Bolsista de iniciação científica. Aluna do $4^{0}$ ano do curso de Enfermagem da Universidade de Maringá - UEM. Maringa - PR. Brasil. E-mail: jessicabatistela@hotmail.com; ${ }^{4}$ Bolsista de iniciação científica. Aluna do $4^{0}$ ano do curso de Enfermagem da Universidade de Maringá - UEM. Maringa - PR. Brasil. E-mail: colucci.andrea@hotmail.com
} 


\section{INTRODUÇÃO}

No Brasil, a atenção à saúde materna e infantil, historicamente, tem sido uma prioridade entre as políticas de saúde, com destaque aos cuidados durante a gestação. A partir de 1970, tal política teve um incremento substancial, devido às elevadas taxas de morbidade e mortalidade materna $\mathrm{e}$ infantil, com ampliação da atenção ao pré-natal, face ao reconhecido impacto e transcendência que esta produz no estado de saúde da mãe e do feto.

A mortalidade infantil é reconhecida como um indicador da condição de vida e de saúde de uma população e constitui uma referência importante por conter informação relevante sobre determinados atributos e dimensões do estado de saúde, bem como do desempenho do sistema de saúde. ${ }^{2}$

Os indicadores de saúde são unidades de medida de uma atividade, ou, ainda, uma medida quantitativa que pode ser usada como um guia para monitorar e avaliar a qualidade assistencial e as atividades de um serviço, que são normalmente apresentados por meio de taxas ou coeficientes. ${ }^{3}$ A qualidade de um indicador depende das propriedades dos dados utilizados em sua formulação, determinada pela frequência de casos, pelo tamanho da população em risco e pela precisão dos sistemas de informação empregados. ${ }^{2}$

Em 2008, a taxa de mortalidade infantil no Brasil era de 17,6 para cada 1.000 crianças nascidas vivas, sendo no Paraná e em Maringá 13,1 e 8,5, respectivamente. 0 óbito infantil, neste caso, normalmente relacionava-se com agravos da gestação que aceleram o nascimento da criança ou levam a complicações no momento do parto. Já em menores de cinco anos, a taxa de mortalidade alcançou 15,40 no estado. ${ }^{4} 0$ óbito, nesta idade possivelmente estava associado a doenças como pneumonia, desnutrição e diarreia, julgadas, na maioria dos casos, como evitáveis.

A taxa de mortalidade descreve a situação de saúde de uma localidade, mas de forma generalizada. No entanto, é importante avaliar qualitativamente os óbitos, buscando detectar as causas evitáveis de óbito, delimitando diagnósticos quanto à rede de assistência em saúde.

Para tal avaliação, Rutstein ${ }^{5}$ apresentou uma lista com 90 causas de óbito que poderiam ser evitadas na existência de um serviço de saúde efetivo. Sabe-se que as estimativas de óbito evitável podem contribuir para a tomada de decisões considerando-se os indicadores de qualidade da atenção à saúde não satisfatórios. ${ }^{6}$ Além de Rutstein, autores como Berenberg, Chalmers ${ }^{5}$ propuseram um novo método para mensurar a qualidade do cuidado, 0 qual abrange casos de doenças preveníveis, as morbidades e as mortes evitáveis. A pergunta norteadora dessa ocorrência é: "Por que eles acontecem?".

Esses autore ${ }^{5}$ classificaram a mortalidade infantil, o óbito por infecções intestinais, a pneumonia em menores de quatro anos, entre outros, como causas de morte evitáveis ou evento sentinela, por apresentarem possibilidades de intervenção, determinadas pela existência de tecnologia apropriada para evitar o aparecimento da doença, a sua progressão e óbito, independentemente do fato de os procedimentos não estarem disponíveis para a maioria da população.

Considerando os eventos supracitados como fatos evitáveis, que o serviço de atenção primária em MaringáPR possui estrutura satisfatória para ações de promoção e prevenção de saúde e que os protocolos de cuidados às gestantes e às crianças estão estruturados na rede nacional de atenção básica, decidiu-se analisar quais óbitos, ocorridos em 2008, entre menores de cinco anos poderiam ter sido evitados no município de Maringá, PR, fornecendo assim embasamento científico para futuras intervenções em saúde.

\section{MÉTODO}

Pesquisa quantitativa, com caráter descritivo, dos 41 óbitos ocorridos em 2008, de crianças menores de cinco anos residentes no município Maringá-PR, que dista aproximadamente $428 \mathrm{~km}$ da capital, Curitiba, de população estimada em 335.512 habitantes. Conta com 973 estabelecimentos de saúde, sendo 26 unidades básicas e 1.161 leitos para internações hospitalares. ${ }^{4}$

Para investigação do óbito em menores de um ano foram utilizados os registros do Comitê de Prevenção do Óbito Infantil e Fetal, nos quais consta um levantamento de dados de todo 0 atendimento de saúde realizado para a gestante e a criança. Além disso, o Comitê realiza, dentro de sua rotina de atuação, a entrevista domiciliar que traz informações não registradas em prontuários para preenchimento total do formulário de investigação do óbito.

0 formulário para a investigação dos óbitos fetais, neonatais ou pós-neonatais compõe-se de dados de identificação da criança, de notificação e investigação do óbito, de características e condições da família e da mãe, da gestação, do nascimento e acompanhamento da criança, da doença que causou a morte, e registros dos serviços de saúde. $^{?}$

Em crianças maiores de um ano e menores de cinco anos, a identificação dos óbitos aconteceu por busca ativa em declarações de óbito (D0) na Secretaria Municipal de Saúde. Foram utilizados os mesmos critérios e meios para avaliação da condição do óbito que o Comitê de Óbito Infantil. Como instrumentos de investigação foram utilizados os prontuários de unidades básicas, serviços de urgência, ambulatórios e de especialidades, e prontuários hospitalares.

Para a análise da evitabilidade dos óbitos, utilizou-se o referencial de Rutstein et al..$^{5}$, precursor da temática em 1976, que classifica, dentre outras causas, as doenças descritas no Quadro 1 como eventos evitáveis na criança. 
Quadro 1 - Lista de doenças/eventos evitáveis relacionados à criança. ${ }^{6}$

\begin{tabular}{|l|c|c|}
\hline \multicolumn{1}{|c|}{ Condiçōes de saúde evitáveis } & CID-9 & Nós críticos da assistência \\
\hline $\begin{array}{l}\text { Outras doenças infecciosas por salmonela como } \\
\text { veículo de contaminação da comida }\end{array}$ & 003 & Prevenção \\
\hline Difteria & 032 & Prevenção/tratamento \\
\hline Tétano & 037 & Prevenção \\
\hline Catapora & 050 & Prevenção \\
\hline Rubéola & 056 & Prevenção \\
\hline Febre amarela & 060 & Prevenção \\
\hline Leucemia mieloide & 205 & Prevenção- exposiçào à radiação \\
\hline Avitaminoses ou outras deficiências nutricionais & $260-269$ & $\begin{array}{c}\text { Prevenção/tratamento (não associados a } \\
\text { neoplasias ou má absorção) }\end{array}$ \\
\hline Anemias & $280-284$ & Tratamento \\
\hline $\begin{array}{l}\text { Meningite bacteriana (Haemophilus influenzae tipo } \\
\text { B, pneumococcus, streptococcus grupo } A,\end{array}$ & 320 & Tratamento \\
Staphylococcus aureus) & & Prevenção \\
\hline Doenças cardiopulmonares & 426 & Tratamento \\
\hline $\begin{array}{l}\text { Infecções respiratórias, influenza, pneumonia, } \\
\text { bronquite }\end{array}$ & $460-466$ & Prevenção \\
\hline Mortalidade infantil (todas as causas) & $760-778$ & \\
\hline
\end{tabular}

As medidas de evitabilidade foram baseadas na lista de causas de mortes evitáveis em menores de cinco anos por intervenções no âmbito do Sistema Único de Saúde do Brasil, publicada em 2007, por Malta et al. ${ }^{6}$; essas intervenções foram classificadas em atividades de prevenção, ações de diagnóstico e tratamento, e outras.

A organização dos dados aconteceu em formato de tabelas de frequência, criadas por meio do programa Statística 8.0.

Este estudo teve aprovação da Secretaria Municipal de Saúde no dia 30/11/2009, do Comitê de Ética em Pesquisa da Universidade Estadual de Maringá sob protocolo 650 em 11/12/2009 e do Comitê de Ética em Pesquisa Estadual sob protocolo 162 de 25/03/2010.

\section{RESULTADOS}

Por meio do Comitê de Investigação do Óbito Infantil do município foram contabilizados 37 óbitos (menores de um ano) no ano de 2008. Ao realizar a busca ativa das D0 das crianças entre um e cinco anos na Secretaria Municipal de Saúde detectaram-se cinco óbitos, totalizando 42 óbitos. No entanto, quando se procedeu a entrevista aos familiares de um dos casos, constatou-se que a criança tinha idade real de cinco anos e quatro meses, contrapondo a idade de quatro anos e sete meses registrada na D0, motivando a exclusão do caso na discussão deste trabalho.

Dos 41 casos investigados, 27 (65,9\%) representaram óbitos neonatais, inferiores a 28 dias, 10 $(24,4 \%)$ pós-neonatais, entre 28 dias e um ano, e quatro $(9,7 \%)$ óbitos de um ano até cinco anos.
Vinte e duas crianças $(53,7 \%)$ eram do sexo masculino e 19 (46,3\%), do sexo feminino. 0 mês de fevereiro destacou-se com $14,6 \%$ dos óbitos, seguido de setembro e dezembro com 12,2\% cada. Segundo o local de ocorrência, 40 (97,6\%) aconteceram em hospitais e um (2,4\%), em domicílio.

$\mathrm{Na}$ Tabela 1, destacam-se a faixa etária das mães de 30 a 34 anos (26,8\%), média de 28,2, variando entre idade mínima de 15 e máxima de 46 anos, desvio-padrão 7,38, 80,5\% com companheiro, grau de escolaridade de 8 a 11 anos $(53,6 \%)$ e raça branca $(80,5 \%)$. Em relação aos hábitos/vícios, quatro $(9,8 \%)$ eram usuárias de drogas ilícitas e três $(7,3 \%)$, tabagistas.

Dos óbitos investigados quase $40 \%$ (16) deles foram de crianças que nasceram com peso inferior a $1.000 \mathrm{~g}$, média de $1.828 \mathrm{~g}$, extremos de $350 \mathrm{~g} \mathrm{e} 4.680 \mathrm{~g}$, também foi observada maior prevalência de parto cirúrgico (Tabela 2).

Ao realizar a distribuição da idade gestacional por financiamento e tipo de parto, observa-se que 18 (43,9\%) óbitos foram de crianças cujas mães foram assistidas no prénatal pela rede privada de saúde e contavam com idade gestacional 22 a 27 semanas, maior entre aquelas que foram atendidas por outros convênios (19,5\%). Já quanto às mães atendidas pelos serviços públicos de saúde, seus filhos nasceram entre a $37^{\mathrm{a}}$ e $41^{\mathrm{a}}$ semana.

Seguindo conceito de Rutstein (1976) sobre evitabilidade ${ }^{5}, 37$ (90,2\%) dos 41 óbitos foram considerados evento sentinela. De modo geral, as causas encontradas em menores de cinco anos foram doenças relacionadas ao período perinatal, malformações congênitas, doenças do sistema respiratório e neoplasias (Tabela 3). 
Os quatro óbitos considerados inevitáveis incluíram situações como: 1) reunião de gêmeos; nesse caso, os dois fetos apresentavam condições incompatíveis com a vida, tinham cardiopatia complexa, compartilhamento de estrutura abdominal e fígado, e foram retirados com ordem judicial, pois a mãe corria risco de morte; 2) um recém-nascido com malformação intrauterina, hidrocefalia, acrescida ainda de síndrome de Down; 3) e uma criança de três anos com síndrome de Menkes, que deriva de uma alteração genética extremamente rara vinculada ao sexo da criança (masculino), a qual desencadeia retardo no desenvolvimento neuromotor, com baixa expectativa de vida.

Dos 37 óbitos evitáveis, 24 (64,9\%) eram neonatais, $10(27,0 \%)$ pós-neonatais e três $(8,1 \%)$, de criança acima de um ano. Segundo as medidas de evitabilidade ${ }^{6}$, observou-se que as atividades de prevenção foram as mais encontradas entre as possibilidades de redução do óbito (22; 59,5\%), seguidas das ações de diagnóstico e tratamento $(13 ; 35,1 \%)$, e outras causas (2; 5,4\%), destacando-se aqui a dependência das mães com drogas ilícitas (Tabela 4).

Tabela 1 - Distribuição dos óbitos em menores de cinco anos, segundo características sociodemográficas da mãe. Maringá, 2008.

\begin{tabular}{lcc}
\hline \multicolumn{1}{|c}{ Variáveis } & N (total=41) & $\%$ \\
\hline Idade & 6 & $14,6 \%$ \\
\hline 15 a 19 anos & 7 & $17,1 \%$ \\
20 a 24 anos & 10 & $24,4 \%$ \\
25 a 29 anos & 11 & $26,8 \%$ \\
30 a 34 anos & 6 & $12,2 \%$ \\
\hline 35 a 39 anos & 1 & $2,4 \%$ \\
\hline 40 a 44 anos & 1 & $2,4 \%$ \\
\hline Acima de 45 anos & & \\
\hline Estado Civil & 33 & $80,5 \%$ \\
\hline Com companheiro & 8 & $19,5 \%$ \\
\hline Sem companheiro & & \\
\hline Escolaridade & 1 & $2,4 \%$ \\
\hline a 3 anos & 6 & $14,6 \%$ \\
\hline 4 a 7 anos & 22 & $53,6 \%$ \\
\hline 8 a 11 anos & 11 & $26,8 \%$ \\
\hline 12 anos ou mais & 1 & $2,4 \%$ \\
\hline Sem informação & & \\
\hline Raça & 33 & $80,5 \%$ \\
\hline Branca & 4 & $9,7 \%$ \\
\hline Parda & 4 & $9,7 \%$ \\
\hline Sem informação & & \\
\hline Renda & 18 & $43,9 \%$ \\
\hline 1 a 3 salários & 6 & $14,6 \%$ \\
\hline a 6 salários & 2 & $4,9 \%$ \\
\hline Acima de 6 salários & 15 & $36,6 \%$ \\
\hline Sem informação & & \\
\hline
\end{tabular}

Fonte: Elaboração Própria

Tabela 2 - Distribuição dos óbitos de menores de cinco anos, segundo parto, idade gestacional e peso ao nascer. Maringá, 2008.

\begin{tabular}{|c|c|c|}
\hline Variáveis & $N($ total $=41)$ & $\%$ \\
\hline \multicolumn{3}{|l|}{ Tipo de Parto } \\
\hline Cesáreo & 23 & $56,1 \%$ \\
\hline Vaginal & 18 & $43,9 \%$ \\
\hline \multicolumn{3}{|l|}{ Idade Gestacional } \\
\hline Menor de 22 semanas & 2 & $4,9 \%$ \\
\hline 22 a 27 semanas & 13 & $31,7 \%$ \\
\hline 28 a 31 semanas & 7 & $17,1 \%$ \\
\hline 32 a 36 semanas & 6 & $14,6 \%$ \\
\hline 37 a 41 semanas & 12 & $29,3 \%$ \\
\hline 42 ou mais & 1 & $2,4 \%$ \\
\hline \multicolumn{3}{|l|}{ Peso ao nascer } \\
\hline Menos de $500 \mathrm{~g}$ & 1 & $2,4 \%$ \\
\hline 500 a $999 g$ & 15 & $36,6 \%$ \\
\hline 1.000 a $1.499 \mathrm{~g}$ & 5 & $12,2 \%$ \\
\hline 1.500 a $2.499 g$ & 6 & $14,6 \%$ \\
\hline 2.500 a $2.999 g$ & 3 & $7,3 \%$ \\
\hline 3.000 a $3.999 \mathrm{~g}$ & 10 & $24,4 \%$ \\
\hline Acima de $4.000 \mathrm{~g}$ & 1 & $2,4 \%$ \\
\hline
\end{tabular}

Fonte: Elaboração própria 
Tabela 3 - Distribuição dos óbitos de menores de cinco anos, segundo causa principal. Maringá, 2008.

\begin{tabular}{lr}
\hline \multicolumn{1}{c}{ CID principal após investigação do Comitê de Óbito } & N (total=41) \\
\hline Acidose não especif. durante o cuidado médico e cirúrgico & $1(2,4 \%)$ \\
Asfixia grave ao nascer & $1(2,4 \%)$ \\
Atresia de esôfago, sem fistula & $1(2,4 \%)$ \\
Comunicação ventriculo atrial discordante & $1(2,4 \%)$ \\
Enterocolite necrotizante & $1(2,4 \%)$ \\
Estenose congênita de valva aórtica & $1(2,4 \%)$ \\
Inalação do conteúdo gástrico & $1(2,4 \%)$ \\
Insuficiência renal congênita & $1(2,4 \%)$ \\
Insuficiência respiratória* & $1(2,4 \%)$ \\
Leucemia mieloide aguda & $1(2,4 \%)$ \\
Malformação especificada do coração & $1(2,4 \%)$ \\
Outras malformações congênitas especificadas & $1(2,4 \%)$ \\
Outras malformações congênitas & $1(2,4 \%)$ \\
PN não especificada & $3(7,3 \%)$ \\
Reunião de gêmeos & $2(4,9 \%)$ \\
RN afetado por afeç̧̃ão materna especifica & $1(2,4 \%)$ \\
RN afetado por corioamnionite & $2(4,9 \%)$ \\
RN afetado por doenças infecciosas da mãe & $1(2,4 \%)$ \\
RN afetado por doenças maternas renais e vias urinárias & $3(7,3 \%)$ \\
RN afetado por gravidez múltipla & $2(4,9 \%)$ \\
RN afetado por incompetência de colo uterino & $1(2,4 \%)$ \\
RN afetado por outras formas de hemorragia & $1(2,4 \%)$ \\
RN afetado por parto precipitado & $2(4,9 \%)$ \\
RN afetado por ruptura de membrana & $3(7,3 \%)$ \\
RN afetado por transtorno materno hipertensivo & $2(4,9 \%)$ \\
RN afetado por uso de drogas que causam dependência na mãe & $1(2,4 \%)$ \\
Sindrome de Menkes* & $1(2,4 \%)$ \\
Síndrome do filho de mãe diabética & $1(2,4 \%)$ \\
Meningococcemia* & $2(4,9 \%)$ \\
\hline
\end{tabular}

* Maiores de um ano

Fonte: Elaboração Própria

Tabela 4 - Distribuição das causas evitáveis, conforme classificação de Malta et al. (2007), por faixa etária de óbito. Maringá, 2008.

\begin{tabular}{|c|c|c|c|}
\hline Causas evitáveis & $\begin{array}{l}\text { Obitos neonatais } \\
(<28 \text { dias })\end{array}$ & $\begin{array}{c}\text { Obitos infantis- } \\
\operatorname{tardios}(>28 d<1 \\
\text { ano) }\end{array}$ & $\begin{array}{c}\text { Obitos de } \\
\text { maiores de } \\
\text { um ano }\end{array}$ \\
\hline \multicolumn{4}{|l|}{ 1. Reduziveis por adequada atenção à mulher na gestação } \\
\hline $\begin{array}{l}\text { Afecções maternas que afetam o feto ou o recém- } \\
\text { nascido }\end{array}$ & 3 & 1 & - \\
\hline $\begin{array}{l}\text { Complicações maternas da gravidez que afetam o } \\
\text { feto ou recém-nascido }\end{array}$ & 13 & 2 & - \\
\hline \multicolumn{4}{|l|}{ 2. Reduziveis por adequada atenção à mulher no parto } \\
\hline $\begin{array}{l}\text { Outras complicações do trabalho de parto ou parto } \\
\text { que afetam o recém-nascido }\end{array}$ & 2 & - & - \\
\hline 3. Reduziveis por adequada atenção ao recém-nascido & 1 & - & - \\
\hline $\begin{array}{l}\text { 4. Reduziveis por ações adequadas de diagnóstico e } \\
\text { tratamento }\end{array}$ & 4 & 6 & 3 \\
\hline 5. Outras causas & 1 & 1 & 0 \\
\hline Total $(n=37)$ & $24(64,9 \%)$ & $10(27,0 \%)$ & $3(8,1 \%)$ \\
\hline
\end{tabular}

Fonte: Elaboração Própria 


\section{DISCUSSÃO}

Julgou-se pertinente citar o caso excluído, pois o dado referente à idade da criança preenchida na D0 é lançado nos sistemas de informação em saúde, neste caso, o Sistema de Informação de Mortalidade (SIM). 0 preenchimento incorreto dos campos dos formulários pode refletir na situação epidemiológica de dada população. As informações epidemiológicas têm sido cada vez mais utilizadas em nível regional e local para análise dos problemas de saúde e de suas causas.

O SIM contém informações sobre o óbito, tais como: causa básica, data, local e município de ocorrência, assim como informaç̃os sobre o falecido (idade, sexo, grau de escolaridade, ocupação e município de residência). Um dos grandes problemas que ainda permeiam as análises de mortalidade no Brasil é 0 sub-registro de óbitos, que tem magnitude expressiva, sobretudo nas regiões norte e nordeste, com predomínio entre os menores de um ano. No entanto, os esforços para o aperfeiçoamento dos sistemas de informação em saúde têm ocorrido de forma progressiva, resultando em crescente cobertura e melhora da qualidade. ${ }^{8}$

As inconsistências do SIM, relacionadas principalmente às variáveis sexo, idade e causa básica, são inferiores a 0,5\% do total de óbitos. ${ }^{9}$ Algumas limitações desse sistema, no entanto, devem ser consideradas, entre elas: o preenchimento incorreto ou incompleto da D0; a revisão deficiente ou inexistente dos dados informados; ausência de retorno das DOs com erros para a unidade informante; codificação imperfeita e digitação terceirizada; ausência de relatórios de inconsistências; e elevado número de óbitos por causas mal definidas. $^{9,10}$

Quanto à representação dos óbitos, o estudo demonstra que a maioria ocorreu em neonatos, ficando consequentemente nesta categoria a maior proporção de evitáveis. Em uma pesquisa realizada em Porto Alegre, com o objetivo de investigar a associação entre óbitos evitáveis e não evitáveis com variáveis sociodemográficas e reprodutivas maternas, destacou-se que a morte precoce tem associação direta com a qualidade da atenção dada à gestante, ao parto e ao recém-nascido, evidenciando a importância de ações de prevenção da mortalidade infantil por meio de uma adequada assistência materno-infantil. ${ }^{11}$

Essa asser tiva se sustenta no âmbito mundial, onde a problemática dos óbitos em menores de cinco anos expia preocupação alargada, com maior porcentagem entre os óbitos neonatais, seguidos de pneumonia e doenças diarreicas, todas classificadas como evitáveis, ou seja, passíveis de prevenção ${ }^{12}$.

Em relação à idade materna que apresenta maior probabilidade de morte em crianças, este estudo demonstrou que os óbitos aconteceram com mães com idade entre 25 e 34 anos, destacando-se o fato de que essa faixa etária também representa o maior número de nascimentos. Há estudos que confrontam esta afirmativa mostrando que o óbito infantil normalmente acontece em mães com idades extremas, menores de 15 anos e maiores de 40.11,13

Um estudo realizado no município de Maringá-PR no período de 1999 a 2006 com o objetivo de caracterizar os óbitos infantis mostrou a idade média das mães de 24,6. Da mesma forma que os resultados apresentados neste estudo, as informações sobre renda foram prejudicadas, pois ainda existe uma porcentagem significativa dos dados ignorados..$^{13}$

Pesquisas revelam que a elevada mortalidade perinatal e neonatal das crianças têm relação direta com baixo poder aquisitivo ou pouca escolaridade das famílias. ${ }^{11,14}$ Tomando-se a característica sócio-econômico-cultural do município em questão, com média de alfabetização populacional de 92,9\%, segundo IBGE ${ }^{15}$, pode-se esperar que a escolaridade das mães seja superior quando comparada à média nacional, e consequentemente, a outros estudos. Isso não desmerece a discussão desta variável, considerando que quanto maior 0 nível de escolaridade, maior seria a informação e, então, a acessibilidade aos serviços de saúde.

0 peso ao nascer constitui eficiente indicador de prognóstico de morte perinatal e destaca-se como o fator mais importante para explicar o efeito das variáveis sociais e biológicas nessa mortalidade. ${ }^{13}$ Outro estudo constatou que os nascidos vivos de baixo peso apresentaram um risco de óbito 44,10 vezes maior que os nascidos vivos de peso normal e uma probabilidade de morte de 69,2 por mil. ${ }^{16}$ Em Maringá, por meio do Programa Bebê de Risco, as crianças com baixo peso são incluídas e acompanhadas continuamente até atingirem um ano de idade com peso adequado e receberem alta.

A prematuridade também contribui para o aparecimento de riscos, agravos e sequelas no processo do desenvolvimento e crescimento infantil, tornando-se necessário prever e considerar tais riscos e prognósticos para que se possa eventualmente instaurar e promover medidas preventivas. ${ }^{17}$

Sobre o tipo de parto, nota-se a predominância do parto cesáreo, caracterizado como uma intervenção cirúrgica que deve ser realizada quando existem riscos para a mãe, feto ou ambos, antes ou no decorrer do trabalho de parto. Este tipo de parto está associado à mortalidade materna e infantil, podendo ocasionar a síndrome de angústia respiratória e prematuridade iatrogênica no recém-nascido, e infecções puerperais, embolia pulmonar, íleo paralítico, hemorragias e reações indesejáveis da anestesia na mãe. Ainda, por realizar a separação da mãe e filho no pós-parto imediato, interfere no estabelecimento do vínculo mãe-bebê e na amamentação precoce e efetiva. ${ }^{18}$

0 coeficiente de cesarianas no Brasil é um dos mais elevados do mundo. De acordo com o Sistema de Informação de Nascidos Vivos, no Estado do Paraná, os índices de cesariana chegaram a 55\% no ano de 2008; no entanto, em Maringá foram realizados $77,1 \%$ dos partos cesáreos contra o valor de 
15\% recomendado pela Organização Mundial de Saúde. Acredita-se que no mundo moderno, muitas mães procuram por um parto programado e com alta tecnologia, não o vendo como um processo fisiológico e natural.

Para a discussão dos resultados referentes ao cruzamento da idade gestacional com o financiamento e tipo de parto, acredita-se que se faz necessário refletir o processo de captação da gestante e qualidade da consulta de pré-natal, independente da característica privada ou pública do serviço. Um estudo com o objetivo de avaliar o atendimento às mães no pré-natal a partir da investigação dos óbitos em menores de cinco anos ocorridos em 2008 em Maringá-PR concluiu que apenas $38,5 \%$ gestantes estudadas tiveram sua primeira consulta de pré-natal no primeiro trimestre; $50,0 \%$ realizaram acima de seis consultas; $73,1 \%$ foram avaliadas quanto ao risco gestacional; apenas $34,6 \%$ dos prontuários tinham registro de informações sobre exames laboratoriais de prénatal, e 50,0\% dos nascimentos ocorreram por parto cesáreo. Além disso, os autores ainda discutem a dificuldade de acesso aos prontuários da rede privada como um fator dificultador na avaliação do atendimento às mães nas consultas. ${ }^{19}$

Diante desse fato, pode-se perceber que o registro completo em prontuário e as informaç̧ões precisas nos sistemas de saúde são essenciais para a consolidação de estudos sobre evitabilidade de óbitos, para, assim, trazer maior validade em seus resultados e análises.

Este estudo mostrou que 90,2\% dos óbitos eram evitáveis, sendo 59,5\% com medidas de prevenção. Vale dizer que não foram encontradas causas de óbito por doenças diarreicas no período estudado, um fator de evolução entre as causas. Um estudo que buscou avaliar o impacto da municipalização na qualidade dos serviços prestados comprovou que a rede ambulatorial em Maringá-PR se expandiu positivamente na década de 80 , com o declínio das mortes evitáveis, principalmente relacionadas às doenças infecciosas intestinais, pelo maior acesso da população aos serviços. ${ }^{20}$

A redução da mortalidade peri e neonatal tem sido mais difícil e lenta que a mortalidade pós-neonatal, já que esta última é mais vulnerável às melhorias globais da condição de vida e às intervenções do setor de saúde. A mortalidade perinatal está relacionada ao atendimento obstétrico e neonatal e ao impacto de programas de intervenção nesta área, pela estreita relação entre a assistência prestada à gestante e ao recém-nascido. $^{14}$

\section{CONSIDERAÇÕES FINAIS}

Este estudo demonstrou que a partir de um evento indesejado, o óbito, pode-se retrospectivamente fazer uma investigação das informaç̧ões coletadas com a família ou em prontuário. Por meio do perfil epidemiológico do óbito, faz-se o levantamento das causas mais frequentes e dos fatores intervenientes e promovem-se informações para discutir medidas de prevenção e promoção de saúde à mãe no prénatal e a criança na puericultura.

Entre o quantitativo de óbitos de menores de cinco anos ainda se encontram, em sua maioria, óbitos neonatais que têm relação direta com a assistência à gestante e ao parto. Portanto, espera-se que as unidades básicas de saúde instituam a avaliação como rotina de suas práticas mediante os casos de óbitos, entendendo que a prevenção de doenças e a promoção de saúde se constituem em ações prioritárias neste nível de atenção.

Espera-se que este estudo contribua para o incentivo de novas pesquisas sobre 0 assunto, reconhecendo algumas limitações como as falhas de registros em prontuários e dificuldade de acesso às informações de clínicas privadas, mesmo com o auxílio do Comitê de Prevenção do Óbito. Diante disso, sugere-se a necessidade de um melhor planejamento dos cuidados pré-natal e da assistência ao parto para que as práticas sejam eficazes e efetivas a todos os grupos de diversas condições econômicas.

\section{REFERÊNCIAS}

1.Ximenes Neto FRG, Leite JL, Fuly PSC, Cunha ICKO, Clemente AS et al. Qualidade da atenção ao pré-natal na Estratégia Saúde da Família em Sobral, Ceará. REBEN. 2008 set./out.; 61(5): 595-602.

2.Organização Pan-americana de Saúde. Indicadores básicos para a saúde no Brasil: conceitos e aplicações. [citado 2009 out. 20]. Brasília: OPAS; 2008. Disponível em < http://tabnet.datasus.gov.br/tabdata/livroidb/2ed/ apresent.pdf $>$.

3.Bittar OJN. Indicadores de qualidade e quantidade em saúde. Rev. adm. saúde. 2001; 3(12): 21-8.

4.Ministério da Saúde (Brasil). Datasus: informações de saúde. Estatísticas Vitais. [citado 2011 nov 19]. Disponível em: < http://uww2.datasus.gov.br/ DATASUS/index.php?area=0205>.

5.Rutstein DD, Berenberg W, Chalmers TC, Child CG 3rd, Fishman AP, Perrin EB. Measuring the quality of medical care: a clinical method. N Engl J Med. 1976; 294(11): 582-8.

6.Malta DC, Duarte EC, Almeida MF, Dias MAS, Morais Neto OL, Moura L, et al. Lista de causas de mortes evitáveis por intervenções do Sistema Único de Saúde do Brasil. Epidemiol. serv. saúde. 2007 out./dez; 16(4): 23344.

7.Ministério da Saúde (Brasil). Secretaria de Atenção à Saúde. Departamento de Ações Programáticas Estratégicas. Manual dos comitês de prevenção do óbito infantil e fetal. Brasília(DF): Editora MS; 2004. 
8.Ministério da Saúde (Brasil). Uma análise da situação da sáude. Evoluç̧ão da mortalidadee no Brasil. [citado em 2012 jul. 17]. Disponível em: $<$ http://portal.saude.gov.br/portal/arquivos/pdf/capitulo3_sb.pdf>.

9.Carvalho DM. Grandes sistemas nacionais de informação em saúde: revisão e discussão da situação atual. Inf. Epidemiol. Sus. 1997; 6(4): v7-46.

10.Amaral TCL. Mortalidade hospitalar na rede SUS: espelho dos óbitos ocorridos na população brasileira. [dissertação], Rio de Janeiro: Instituto de Medicina Social da Universidade do Estado do Rio de Janeiro; 2002.

11.Jobim R, Aerts D. Mortalidade infantil evitável e fatores associados em Porto Alegre, Rio Grande do Sul, Brasil, 2000-2003. Cad. saúde pública. 2008 jan.; 24(1): 179-87.

12.World Health Organization - WHO. Child Health Epidemiology Reference Group (CHERG) estimates of the percent distribution of under-five deaths by cause available in the WHO, World Health Report 2005; estimates of total under-five deaths for the year 2006 based on the work of the UNICEF/WHO/World Bank/UN Population Division Child Mortality Estimation Group (available in the forthcoming The State of the World's Children 2008) [citado 2009 dez 11]. Disponível em < http://www.unicef.org/media/ files/Under_five_deaths_by_cause_2006_estimates3.doc $>$.

13.Nabhan SS, Oliveira RZ. Óbitos infantis, características maternas e de assistência em município da região noroeste do Paraná, Brasil, 1999 a 2006. Acta Sci. Health Sci. 2009; 31(1): 71-6.

14.Lansky S, Franca E, Lael MC. Mortalidade perinatal e evitabilidade: revisão da literatura. Rev. saúde pública. 2002; 36(6): 759-72.

15.Instituto Brasileiro de Geografia e Estatística. Censo Demográfico 2000. Atlas do Desenvolvimento Humano. [citado em 2011 nov. 20]. Disponível em: <http://www.ibge.gov.br>.

16.Maran E, Uchimura TT. Mortalidade Neonatal: fatores de risco em um município no sul do Brasil. Rev. eletrônica enferm. 2008; 10(1): 29-38.

17. Ramos HÂC, Cuman RKN. Fatores de risco para prematuridade: pesquisa documental. Esc. Anna Nery Rev. Enferm. 2009; 13(2): 297-304.

18. Rattner D. A epidemiologia na avaliação da qualidade: uma proposta. Cad. saúde pública.1996; 12 Supl 2: S21-32.

19.Jodas DA, Scochi MJ, Moura MB, Tiwata MZ. Atendimento à crianças e às mães: investigação do óbito evitável em menores de cinco anos. Rev. gaúch. enferm. Porto Alegre(RS) 2011 dez; 32(4): 669-75.

20.Scochi MJ. Evolução da mortalidade por causas evitáveis e expansão dos recursos municipais de saúde em Maringá(PR). Rev. saúde pública. 1999; 33(2): 129-36

\section{NOTA}

`Texto extraído da Dissertação de Mestrado intitulada 'Mortalidade evitável em menores de cinco anos: evento sentinela da qualidade dos serviços primários em Maringá-PR' 